\title{
AVALIAÇÃO AMBIENTAL DE LAGOAS NATURAIS E ARTIFICIAIS NO MUNICÍPIO DE BURITICUPU, MA
}

Karoline Lopes Nicasio ${ }^{1}$; Francisco Maciel B. dos Santos ${ }^{1}$; Kaires Mayane A. da Silva"; Jesuino da Silva C. Martins ${ }^{2}$; Reinaldo Lucas Cajaíba ${ }^{2}$

${ }^{1}$ Estudante do Curso de Licenciatura em Biologia do Instituto Federal de Educação, Ciência e Tecnologia do Maranhão, Campus Buriticupu Buriticupu-Brasil ${ }^{2}$ Laboratório de Ecologia e Conservação, Instituto Federal de Educação, Ciência e Tecnologia do Maranhão, Campus Buriticupu. reinaldocajaiba@hotmail.com

\section{Recebido em: 06/04/2019 - Aprovado em: 10/06/2019 - Publicado em: 30/06/2019 DOI: 10.18677/EnciBio_2019A136}

RESUMO

Lagoas são características comuns em diferentes paisagens que contribuem com a biodiversidade regional da água doce. A conservação e preservação desses ecossistemas é de fundamental importância para a manutenção dos hábitats das espécies. O presente estudo teve como objetivo avaliar a qualidade ambiental de lagoas naturais e artificiais do município de Buriticupu, Maranhão, através do uso de protocolo de diversidade de habitats. Ao todo, foram avaliadas 29 lagoas entre naturais e artificiais. Verificou-se na aplicação do protocolo alterações de origem antrópicas em $82,8 \%$ das lagoas amostradas (24 lagoas), variando entre moderadas (11 lagoas) a severas (13 lagoas). Nota-se que a qualidade ecológica das lagoas está fortemente afetada por atividades de pastagens, agroflorestas, agricultura, pesca e residências. O protocolo utilizado demonstrou ser uma boa ferramenta de monitoramento, capaz de identificar os impactos causados na água regional.

PALAVRAS-CHAVE: Monitoramento; Qualidade da água; Sistemas lênticos.

\section{ENVIRONMENTAL EVALUATION OF NATURAL AND ARTIFICIAL PONDS IN THE MUNICIPALITY OF BURITICUPU, MA}

\begin{abstract}
Ponds are common features in different landscapes that contribute to the regional freshwater biodiversity. The conservation and preservation of these ecosystems is of fundamental importance for the maintenance of the species' habitats. The present study had as objective to evaluate the environmental quality of natural and artificial ponds of the municipality of Buriticupu, Maranhão, through the use of habitat diversity protocol. In all, 29 ponds between natural and artificial were evaluated. In the application of the protocol, changes in anthropic origin occurred in $82.8 \%$ of the sampled lagoons (24 ponds), ranging from moderate (11 ponds) to severe (13 ponds). It is noted that the ecological quality of the lagoons is strongly affected by pasture, agroforestry, agriculture, fisheries and residences. The protocol used proved to be a good monitoring tool capable of identifying the impacts caused in the regional water.
\end{abstract}

KEYWORDS: Monitoring; Water quality; Lentic systems. 


\section{INTRODUÇÃO}

Pressões antrópicas sobre os ecossistemas naturais têm levado a uma intensa remoção da cobertura vegetal natural para a implementação de pastagens, rodovias, urbanização, construção de barragens, processos de eutrofização (CAJAIBA et al., 2018), e por consequente, tem gerado grandes impactos e até mesmo o esgotamento dos recursos hídricos (HILL et al., 2016). Essas ações têm resultado em desmatamentos em áreas de mata ciliar, além de afetar a qualidade dos corpos d'água sobre a comunidade biológica nela existente (THORNHILL et al., 2017), levando ao declínio dos serviços ecossistêmicos (SHWARTZ et al., 2014).

Os ecossistemas lênticos correspondem a ecossistemas de águas paradas e apresentam um papel importante como recurso natural para organismos aquáticos e terrestres, variando de habitats efêmeros a corpos de água permanentes, abrangendo lagos, lagoas, pântanos, charcos, entre outros (HILL et al., 2015). É relativamente recente que as lagoas foram amplamente reconhecidas como importantes habitats de água doce que suportam a biodiversidade aquática (HASSALL; ANDERSON, 2015; THORNHILL et al., 2017). A importância ecológica está relacionada à biodiversidade regional da flora e fauna e da elevada proporção de espécies raras e únicas (DAVIES et al., 2008; HEINO et al., 2017). Dessa forma, a conservação das lagoas é essencial para a manutenção das espécies, que de alguma forma, podem não sobreviver em habitats aquáticos mais permanentes (HILL et al., 2018). Além disso, conhecer as características paisagísticas das lagoas e compreender a relevância ecológica desses ambientes pode até apoiar a tomada de decisões e a gestão/recuperação robusta de ecossistemas em perigo, no âmbito da necessidade de metodologias de avaliação rápidas, padronizadas e econômicas (GODFRAY et al., 1999), justificando assim, a realização de estudos nesses ambientes.

Apesar de sua importância para os ecossistemas, estudos realizados em lagoas são negligenciados (MOUTINHO, 2016). Particularmente para o estado do Maranhão, estudos estão ainda em estágio embrionário. Diante deste cenário, tornase de fundamental importância o monitoramento das alterações ambientais, seja realizado por métodos físico-químicos usuais, organismos bioindicadores ou por meio de ferramentas auxiliares, como os protocolos de avaliação rápida (BRITO et al., 2016). A manutenção sustentável dos recursos hídricos depende de bons instrumentos de planejamento, proteção e utilização dos recursos naturais, uma vez que qualidade da água depende de como os recursos estão sendo explorados (BRIERS et al., 2014).

Neste contexto, este estudo buscou avaliar a qualidade ambiental de lagoas naturais e artificiais no município de Buriticupu - MA, localizado na Amazônia Legal Maranhense por meio de um Protocolo de diversidade de habitats proposto por Baker et al. (1997) e USEPA (2007), adaptado e modificado para ecossistemas semi-lênticos e lênticos.

\section{MATERIAL E MÉTODOS}

Localização da área de estudo

O presente estudo foi desenvolvido no município de Buriticupu - MA (Figura 1). A extensão territorial do município é de $2.545,44 \mathrm{~km}^{2}$ e uma população estimada em de 71.723 habitantes (IBGE, 2018). O relevo é constituído por formações de tabuleiros, separados em faixas por drenos e grotões, situado a uma altitude de $200 \mathrm{~m}$ acima do nível do mar (LIMA et al., 2018). O clima da região é equatorial quente e úmido, uma subdivisão do clima tropical. Apresenta duas estações bem ENCICLOPÉDIA BIOSFERA, Centro Científico Conhecer - Goiânia, v.16 n.29; p. 1773 
definidas: o período chuvoso, de dezembro a junho e o período de estiagem, de julho a novembro. A temperatura média anual varia de 25 a $27^{\circ} \mathrm{C}$; a umidade relativa do ar é de $80 \%$, em média, enquanto os índices pluviométricos estão entre 1.800 e $2.000 \mathrm{~mm}$ (CAJAIBA et al., 2019). Encontra-se inserido em sua totalidade no ambiente geológico conhecido como Bacia Sedimentar do Pindaré, que atinge $12,40 \%$ das Bacias do Estado do Maranhão.

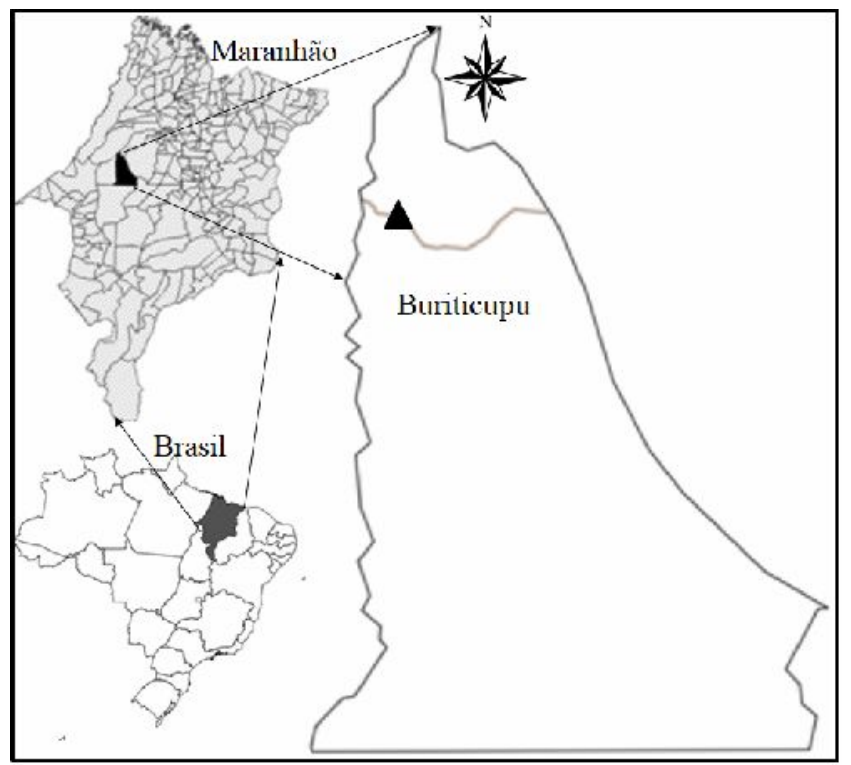

FIGURA 1. Localização geográfica do município de Buriticupu, Maranhão.

\section{Amostragem}

A seleção das lagoas se deu através do uso de imagens de satélites e experiência de campo dos pesquisadores envolvidos. Assim, foram selecionadas 29 lagoas em distintas localidades do município, sendo 20 lagoas naturais e 9 artificiais. Os trabalhos de campo ocorreram entre os meses de agosto a dezembro de 2018.

A caracterização ambiental foi realizada através da verificação in loco dos principais impactos que incidem sobre os locais analisados. Para isso, foi utilizado o protocolo de diversidade de habitats proposto por Baker et al. (1997) e USEPA (2007) e adaptado para ambientes lênticos e semi-lênticos, conforme proposto por Molozzi et al., (2011). A versão do protocolo disponível no site do Laboratório de Ecologia de Bentos-UFMG foi adaptada (http://labs.icb.ufmg.br/benthos/index arquivos/pdfs_pagina/2011/reservatorio.pdf). O protocolo baseia-se em elementoschave para avaliação em ecossistemas (i) avaliação da região litorânea, bosque e sub-bosque ribeirinho em um transecto de $25 \mathrm{~m}$ de observação; (ii) descrição das características ripárias e do litoral em todas as estações de amostragem.

Cada lagoa foi dividida em pontos $(1-5)$ dependendo do seu tamanho, para que assim fosse percorrida toda a sua área ( $25 \mathrm{~m}$ por ponto). Além disso, no momento das visitas, foi realizada uma entrevista com os proprietários e/ou moradores para identificar os principais usos locais de cada lagoa (recreação/ lazer, bebedouro de gado, criação de peixes), bem como identificar se a lagoa era natural ou artificial.

\section{RESULTADOS E DISCUSSÃO}

De acordo com (IBGE, 2010), as principais atividades econômicas no município são a produção extrativista vegetal, pecuária e fruticultura. Porém, 
ultimamente, atividades como a monocultura de eucaliptos e soja tem se expandido pelo município. Segundo Cajaiba et al. (2019), o município Buriticupu, perdeu 97\% da cobertura vegetal nativa.

A aplicação do protocolo evidenciou alterações de origem antrópicas em $82,8 \%$ das lagoas amostradas (24 lagoas), variando de moderadas (11 lagoas) a severas (13 lagoas). Nessas lagoas a qualidade do ambiente está fortemente afetada por atividades de pastagem, monocultura de eucalipto (Eucalyptus sp.) e soja (Glycine max), pesca (tanques de rede) e residências (Figura 2A-B).

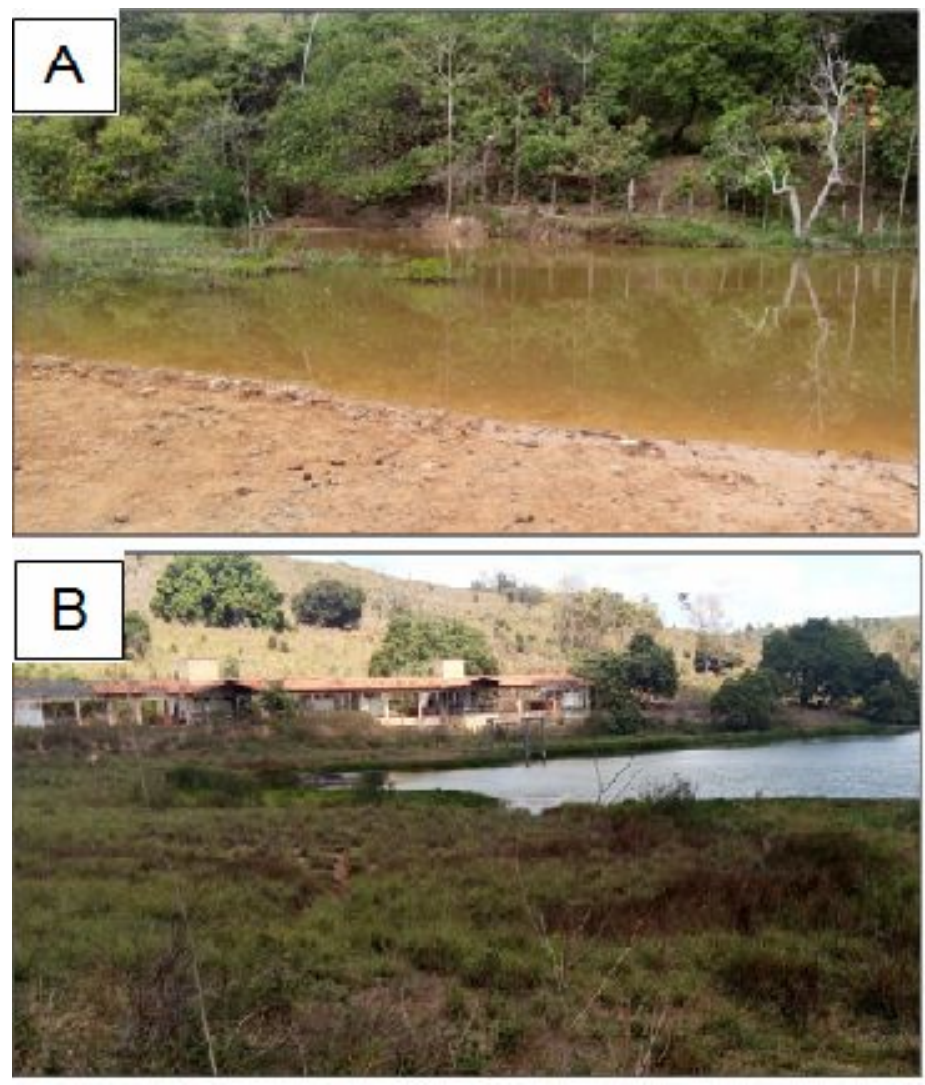

FIGURA 2. Impactos ambientais registrados em lagoas com retirada de mata ciliar no entorno (A) e construção de residências nas proximidades (B).

De acordo com Thornhill et al. (2017), do ponto de vista ambiental, a ocupação urbana gera alterações drásticas nas condições naturais dos sistemas fluviais. Vale destacar que o município de Buriticupu é constituído predominantemente por pequenas propriedades rurais, e as lagoas estão inseridas em seus usos, que consistem em agricultura e pecuária. Verificou-se que das 20 lagoas naturais avaliadas, $60 \%$ delas (12 lagoas) são usadas exclusivamente para criação de gado; 25\% (05 lagoas) para criação de peixes, e 15\% (03 lagoas) para recreação, como o banho. Enquanto nas lagoas artificiais, 66\% (06 lagoas) são destinadas para gado e 34\% (03 lagoas) para criação de peixes em escala comercial (Figura 3).

Áreas complexas apresentam dificuldades de reflorestamento, especialmente se estão ocupadas por pequenas propriedades. Pois neste caso, em geral, a restauração das funções ecológicas e a eficaz conservação da biodiversidade é feita considerando-se os sítios particulares, como: áreas ciliares, zonas-tampão ao entorno das florestas remanescentes, corredores ecológicos, áreas com processo de erosão e as encostas, entre outras (SILVA, 2018). 


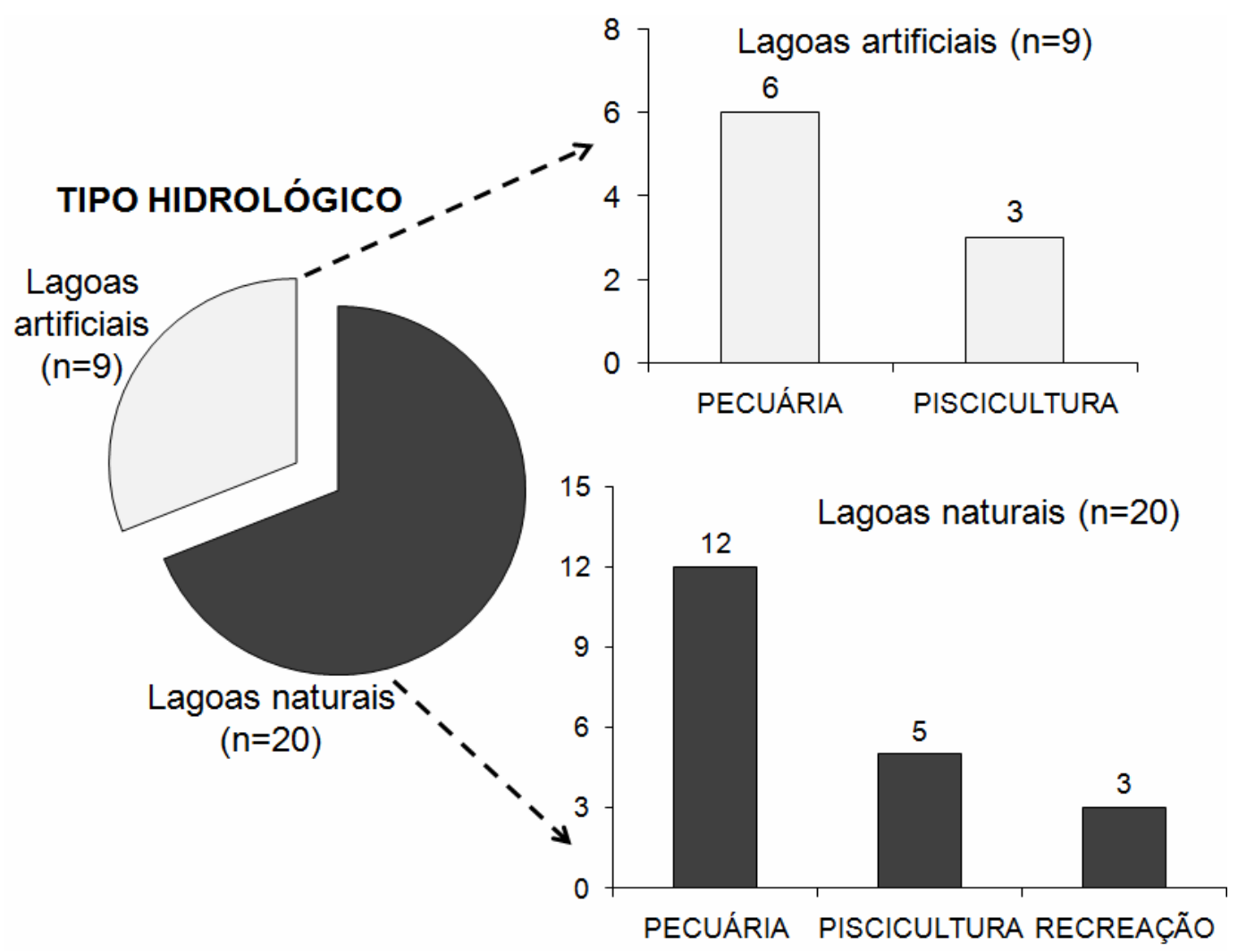

FIGURA 3. Distribuição das lagoas de acordo com o seu tipo hidrológico e uso.

Em 17,2\% das lagoas apresentaram alterações antrópicas em pequeno grau, na qual a vegetação ripária foi mantida, sendo possível a visualização de macrófitas aquáticas. Os poucos distúrbios observados nestas lagoas era o uso de moradores para banho e a pesca em pequena escala (Figura 4). Para Freitas et al. (2013), preservar as características de qualidade da água age garantindo a sobrevivência das espécies aquáticas e consequentemente terrestres nos arredores do curso hídrico, além de permitir seu uso para consumo humano e atividades agroindustriais, e que devido à qualidade garantem um melhor desenvolvimento social, melhorando índices de saúde e qualidade de vida.

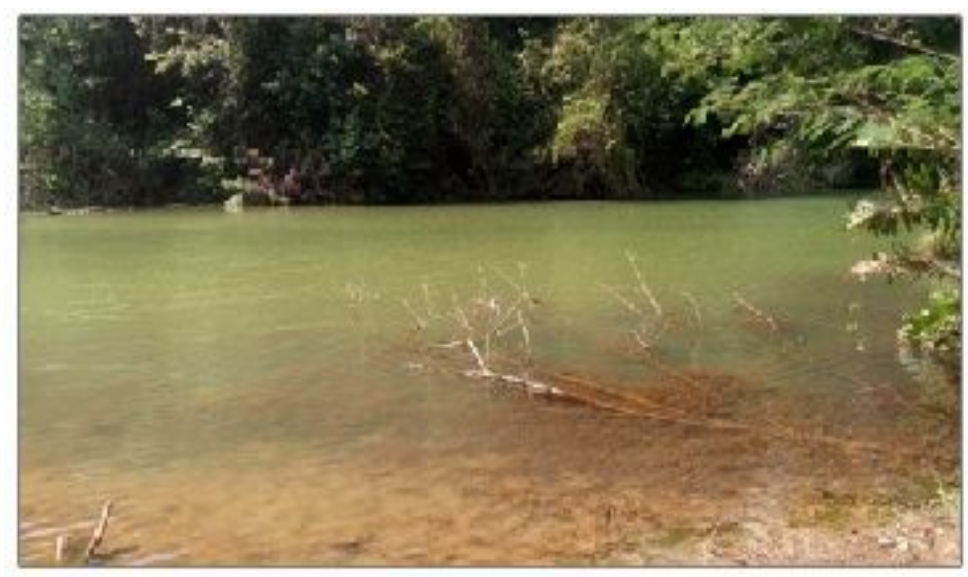

FIGURA 4. Lagoa natural de baixo distúrbio, com vegetação ripária ao entorno, água clara e presença de macrófitas aquáticas. 
Em 27,6\% das lagoas pesquisadas, foi observado solo sem cobertura no entorno, $45,1 \%$ têm cobertura esparsa e $27,3 \%$ contínua. Das lagoas naturais, 38\% não apresentaram dossel, enquanto nas artificiais $55,5 \%$ não apresentam. Além disso, $100 \%$ das lagoas tanto naturais como artificiais apresentaram alguma porcentagem de área queimada, isto indica que na maioria das lagoas são mais numerosos os impactos relacionados à retirada da mata ciliar (Figura 5A-B, Tabela 1).
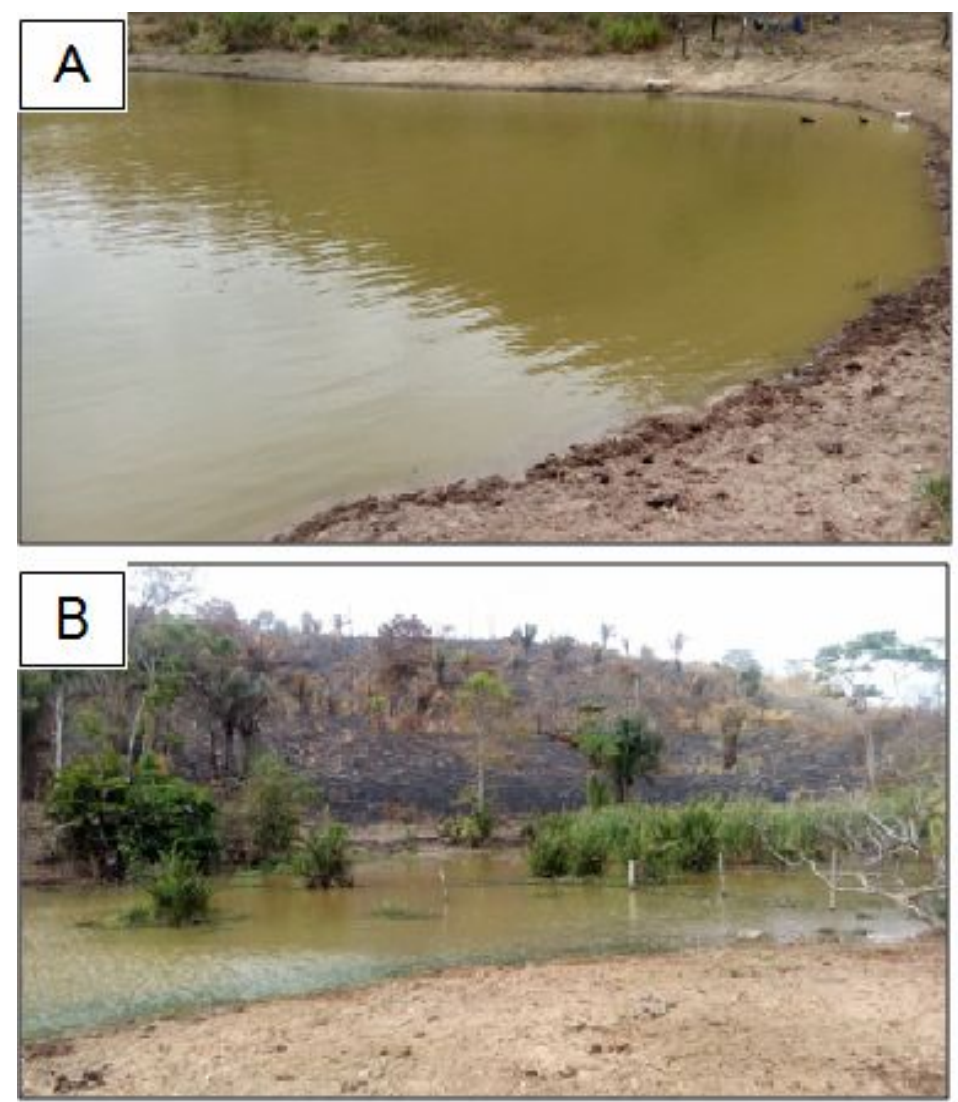

FIGURA 5. Lagoa natural usada para criação de gado. Área ao entorno totalmente desmatada $(A)$ e porção área queimada $(B)$

A ausência deste tipo de vegetação está relacionada ao uso excessivo do solo para pastagens e agricultura. Estudos revelam a importância da mata ciliar no entorno dessas regiões através da constatação de que, em nascentes com vegetação natural remanescente, a qualidade da água mostrou-se melhor que naquelas com entornos agrícolas (DONADIO et al. 2005). De acordo com Hill et al. (2018), a mata ciliar é essencial para o meio ambiente, tendo como uma de suas mais importantes atribuições a manutenção da qualidade da água, estabilidade dos solos, regularização dos ciclos hidrológicos e conservação da biodiversidade.

TABELA 1. Caraterização ambiental das lagoas estudadas no município de Buriticupu, MA

\begin{tabular}{|c|c|c|c|c|}
\hline \multicolumn{5}{|c|}{ Zona ripária } \\
\hline Muito densa (>75\%) & $\begin{array}{c}\text { Densa (40- } \\
75 \%)\end{array}$ & $\begin{array}{c}\text { Moderada } \\
(10-40 \%)\end{array}$ & $\begin{array}{c}\text { Esparsa } \\
(<10 \%)\end{array}$ & $\begin{array}{c}\text { Ausente } \\
(0 \%)\end{array}$ \\
\hline 0 & 2 & 6 & 15 & 6 \\
\hline \multicolumn{5}{|c|}{ Dossel $(>5 \mathrm{~m})$} \\
\hline 0 & $10 \%$ & $15 \%$ & $20 \%$ & $>20 \%$ \\
\hline
\end{tabular}

ENCICLOPÉDIA BIOSFERA, Centro Científico Conhecer - Goiânia, v.16 n.29; p. 1777 


\begin{tabular}{|c|c|c|c|c|}
\hline 12 & 3 & 11 & 3 & 0 \\
\hline \multicolumn{5}{|c|}{ Substrato de fundo } \\
\hline Areia fina & $\begin{array}{c}\text { Areia } \\
\text { grossa }\end{array}$ & Silte + Argila & $\begin{array}{l}\text { Detritos } \\
\text { foliares }\end{array}$ & $\begin{array}{l}\text { Matéria } \\
\text { orgânica }\end{array}$ \\
\hline 3 & 10 & 2 & 4 & 10 \\
\hline \multicolumn{5}{|c|}{ Atividades que caracterizam os distúrbios no ecossistema } \\
\hline Residencial & Recreação & Agricultura & Indústria & Ecossistema \\
\hline 4 & 1 & 19 & 4 & 1 \\
\hline \multicolumn{5}{|c|}{ Distúrbio humano } \\
\hline Ausente & Baixo & Moderado & \multicolumn{2}{|c|}{ Alto } \\
\hline 2 & 4 & 9 & \multicolumn{2}{|c|}{14} \\
\hline \multicolumn{5}{|c|}{ \% de área queimada } \\
\hline $0 \%$ & $5-10 \%$ & $10-25 \%$ & $25-50 \%$ & $>50 \%$ \\
\hline 2 & 2 & 19 & 6 & 0 \\
\hline \multicolumn{5}{|c|}{ Avaliação qualitativa de macrófitas aquáticas } \\
\hline Emergentes & Submersas & Flutuantes & \multicolumn{2}{|c|}{ Ausente } \\
\hline 5 & 4 & 11 & \multicolumn{2}{|c|}{9} \\
\hline \multicolumn{5}{|c|}{ Biofilme na superfície } \\
\hline Ausente & Algas & Óleo & \multicolumn{2}{|c|}{ Espuma } \\
\hline 22 & 4 & 1 & \multicolumn{2}{|c|}{2} \\
\hline \multicolumn{5}{|c|}{ Substrato dominante } \\
\hline Lama & Pedregoso & Areia grossa & \multicolumn{2}{|c|}{ Rochoso } \\
\hline 17 & 2 & 10 & \multicolumn{2}{|c|}{0} \\
\hline
\end{tabular}

Observou-se ainda que lagoas destinadas à criação de gado têm uma porcentagem maior de queimadas, devido à necessidade da retirada de outros tipos de espécies para a inclusão da pastagem. A qualidade da água é prejudicada pela concentração de cinzas, além da deposição de sedimentos desprendidos do solo, decorrentes da erosão, para o fundo dos corpos d'água. A prática do uso do fogo, em curto espaço de tempo, tende a modificar as características do solo, a partir da retirada da cobertura vegetal ocorre a perda de sedimentos, de matéria orgânica e de nutrientes. O fogo age também sobre o estrato arbóreo, influenciando, sobretudo, a redução do porte das árvores e o aumento da sua tortuosidade, da composição florística e estrutura da vegetação (GUIMARÃES et al., 2014; ROCHA, 2018).

No geral, as águas das lagoas apresentaram algum nível de turbidez, no entanto, não apresentaram nenhum odor ou oleosidade aparente (Figura 6). Os substratos de fundo eram compostos principalmente, de areia grossa e matéria orgânica, seguidos de detritos foliares (Tabela 1). 


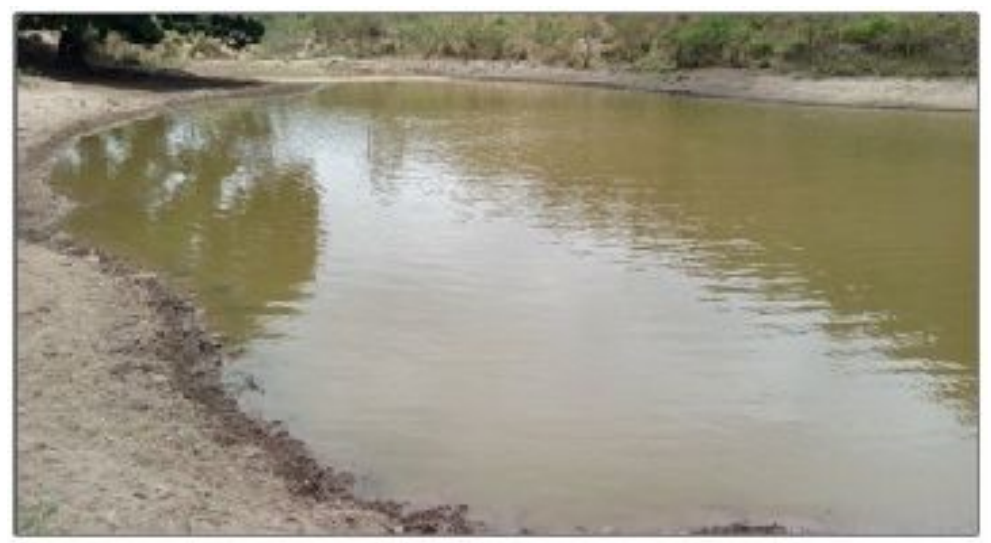

FIGURA 6. Lagoa natural com água turva e sem vegetação ripária.

De acordo com Barros et al. (2017), diferentes substratos são fundamentais para a manutenção do ecossistema aquático e de sua biota. Este mesmo autor afirma que a maior variedade e/ou proporção de substratos em potencial, disponibilizam diferentes nichos, refúgios, alimentos e locais de desova para peixes, macroinvertebrados bentônicos e perifíton, proporcionando incrementos na diversidade biológica.

Notou-se em diversas lagoas pesquisadas, a perda do solo por meio de processos erosivos, reflexo direto da retirada da vegetação ripária para áreas de pastagem. Foram verificadas margens com desmoronamentos e solos expostos ocasionados pela erosão de áreas desmatadas (Figura 7). A retirada da vegetação ripária contribui para o aumento e aceleração de processos erosivos, aumentando também o assoreamento no corpo d'água. A estabilidade das margens evita que ocorra o assoreamento dos cursos d'água garantindo a qualidade da água (FRANCESCHI, 2016; BARBOSA, 2017).
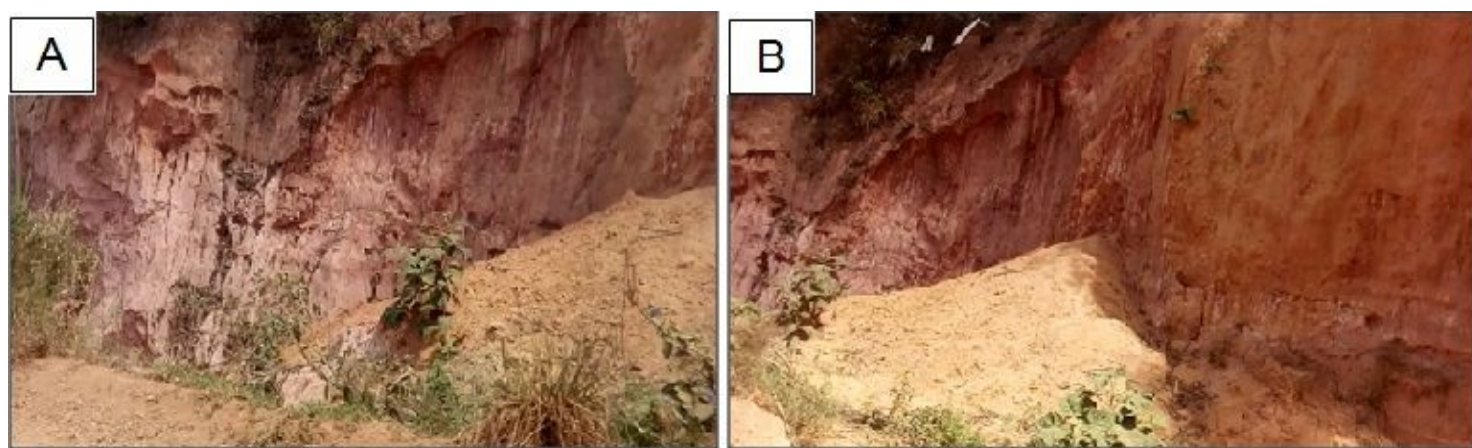

FIGURA 7. Processo erosivo acelerado nas margens de lagoa natural do lado esquerdo $(A)$ e direito $(B)$.

\section{CONCLUSÃO}

O acompanhamento dos corpos d'água por meio da aplicação do protocolo de diversidade de hábitats revelou o estado em que esses ecossistemas se encontram diante da ação antrópica severa encontrada na maioria das lagoas. Diversos tipos de impactos ambientais relacionados a retirada da estrutura natural desses corpos hídricos foram observados, tais como o uso intensivo do solo por pastagens e agricultura, ausência da vegetação ciliar, processos erosivos, e forte presença de população humana ao entorno.

Diante dos impactos ambientais encontrados no município, sentiu-se a carência de investimentos voltados a educação ambiental que vise sensibilizar os 
moradores da região sobre a importância da qualidade ecológica dessas lagoas para a população e gerações futuras.

\section{AGRADECIMENTOS}

Os autores agradecem ao Instituto Federal do Maranhão, campus Buriticupu pelo apoio logístico. KLN agradece ao CNPQ pela bolsa de Iniciação Científica e RLC agradece ao CNPQ pela bolsa de Pós-Doutorado (Processo no 153480/20181).

\section{REFERÊNCIAS}

BAKER, J.R.; PECK, D.V.; SUTTON, D.W. Environmental Monitoring and Assessment Program Surface Waters: Field Operations Manual for Lakes. EPA/620/R-97/001.U.S. Environmental Protection Agency. Corvallis, Oregon. 37p, 1997.

BARBOSA, P.N. Aplicação do índice de sustentabilidade de bacia hidrográfica (WSI) na sub-bacia do Rio Mandu, Minas Gerais. 2017. 62 f. Trabalho de conclusão de curso (Engenharia Ambiental) - Universidade Estadual Paulista Júlio de Mesquita Filho, Instituto de Geociências e Ciências Exatas, 2017.

BARROS, I.T.; CECCON-VOLL, J.; SEREIA, D.O.; BENEDITO, E. Avaliação ambiental e ecológica de riachos neotropicais do alto rio Paraná. Revista da Universidade Vale do Rio Verde, v. 15, n. 2, p.783-790, 2017. Disponível em http://periodicos.unincor.br/index.php/revistaunincor/article/viewFile/3191/pdf_740

BRIERS, A.R. Invertebrate Communities and Environmental Conditions in a Series of Urban Drainage Ponds in Eastern Scotland: Implications for Biodiversity and Conservation Value of SUDS. Clean Soil Air Water, v. 42, n. 2, p. 193-200, 2014. Disponível em https://doi.org/10.1002/clen.201300162

BRITO, M.T.S.; NASCIMENTO FILHO, S.L.; VIANA, G.F.S.; MELO JÚNIOR, M. Aplicação De Um Protocolo De Avaliação Ambiental Rápida Em Dois Reservatórios Do Semiárido Brasileiro. Brazilian Journal of Aquatic Science and Technology, p. 781-793, 2016. Disponível em https://siaiap32.univali.br/seer/index.php /bjast/article/view/6992

CAJAIBA, R.L.; PEREIRA, K.S.; MARTINS, J.S.C.; SOUSA, E.S.; SILVA, W.B. Megasoma actaeon (Linnaeus) (Scarabaeidae: Dynastinae): first record for Maranhão state, northeastern Brazil. Scientia Amazonia, v. 8, p. 13-16, 2019. Disponível em http://scientia-amazonia.org/wp-content/uploads/2018/12/v.8-n.1CB13-CB16-2019.pdf

CAJAIBA, R.L.; PÉRICO, E.; SILVA, W.B.; VIEIRA, T.B.; DALZOCHIO, M.S.; BASTOS, R.; CABRAL, J.A.; SANTOS, M. How informative is the response of Ground Beetles' (Coleoptera: Carabidae) assemblages to anthropogenic land use changes? Insights for ecological status assessments from a case study in the Neotropics. Science of the Total Environment, v. 636, p. 1219-1227, 2018. Disponível em https://doi.org/10.1016/j.scitotenv.2018.04.392

DAVIES, B.R.; BIGGS, J.; WILLIAMS, P.; WHITFIELD, M.; NICOLET, P.; SEAR, D.; BRAY, S.; MAUND, S. Comparative biodiversity of aquatic habitats in the European 
agricultural landscape. Agriculture, Ecosystems and Environment, v. 125, p. 1-8, 2008. Disponível em https://doi.org/10.1016/j.agee.2007.10.006

DONADIO, N. M. M.; GALBIATTI, J. A.; PAULA, R. C. Qualidade da água de nascentes com diferentes usos do solo na bacia hidrográfica do córrego Rico, São Paulo, Brasil. Engenharia Agrícola, v. 24, n. 1, p. 115-125, 2005. Disponível em http://www.scielo.br/pdf/eagri/v25n1/24877.pdf

FRANCESCHI, C.G. A influência do uso e cobertura da terra nos parâmetros da qualidade de água na Bacia hidrográfica do Arroio do Meio-RS. 2017. Trabalho de Conclusão de Curso (Graduação em Geografia) - Universidade Federal Da Fronteira Sul, Erechim, 2016.

FREITAS, E. P.; MORAES, J. F. L.; PECHE FILHO, A.; STORINO, M. Indicadores ambientais para áreas de preservação permanente. Revista Brasileira de Engenharia Agrícola e Ambiental, v. 17, n. 4, p. 443-449, 2013. Disponível em http://dx.doi.org/10.1590/S1415-43662013000400013.

GODFRAY, H.C.J.; LEWIS, O.T.; MEMMOTT, J. Studying insect diversity in the tropics. Philosophical Transations on the Royal Society B, v. 354, p. 1811-1824, 1999. Disponível em doi: 10.1098 / rstb.1999.0523.

GUIMARÃES, P.P.; SOUZA, S.M.; FIEDLER, N.C.; SILVA, A.G. Análise dos impactos ambientais de um incêndio florestal. Agrarian Academy, v. 1, n. 1; p. 39, 2014. Disponível em http://www.conhecer.org.br/Agrarian\%20Academy/ 2014a/analise\%20dos\%20impactos.pdf

HASSALL, C.; ANDERSON, S. Storm water ponds can contain comparable biodiversity to unmanaged wetlands in urban areas. Hydrobiologia, v. 745, p. 137149, 2015. Disponível em https://doi.org/10.1007/s10750-014-2100-5

HEINO, J.; BINI, L.M.; ANDERSSON, J.; BERGSTEN, J.; BJELKE, U.; JOHANSSON, F. Unravelling the correlates of species richness and ecological uniqueness in a metacommunity of urban pond insects. Ecological Indicators, v. 73, p. 422-43, 2017. Disponível em https://doi.org/10.1016/j.ecolind.2016.10.006

HILL, M.J.; HASSALL, C.; OERTLI, B.; FAHRIG, L.; ROBSON, B.J.; BIGGS, J.; SAMWAYS, J.M.; USIO, N.; TAKAMURA, N.; KRISHNASWAMY, J.; WOOD, P.J. New policy directions for global pond conservation. Conservation Letters, v. 11, n. 5, 2018. Disponível em https://doi.org/10.1111/conl.12447

HILL, M.J.; MATHERS, K. L.; WOOD, P.J. The aquatic macroinvertebrate biodiversity of urban ponds in a medium-sized European town (Loughborough, UK). Hydrobiologia, v. 760, n. 1, p 225-238, 2015. https://doi.org/10.1007/s10750-0152328-8

HILL, M.J.; RYVES, D.B.; WHITE, J.C.; WOOD, P.J. Macroinvertebrate diversity in urban and rural ponds: Implications for freshwater biodiversity conservation. Biological Conservation, p 50-59, 2016. https://doi.org/10.1016/j.biocon. 2016.06.027 
IBGE- Instituto Brasileiro de Geografia e Estatística . Censo demográfico. 2010.

IBGE- Instituto Brasileiro de Geografia e Estatística. População estimada: IBGE, Diretoria de Pesquisas, Coordenação de População e Indicadores Sociais, Estimativas da população residente com data de referência $1^{\circ}$ de julho de 2018.

LIMA, J.S.; CAJAIBA, R.L.; MARTINS, J.S.C.; PEREIRA, K.S.; SOUSA, E.S. Educação ambiental em resíduos sólidos em escolas no município de Buriticupu-MA. Scientia Amazonia, v. 7, p. 122-127, 2018. Disponível em http://scientiaamazonia.org/wp-content/uploads/2017/06/v6-n3-11-16-2017.pdf

MOLOZZI, J.; FRANÇA, J.S.; ARAUJO, T.L.A.; VIANA, T.H.; HUGHES, R.M.; CALLISTO, M. Diversidade de habitats físicos e sua relação com macroinvertebrados bentônicos em reservatórios urbanos em Minas Gerais. Iheringia, v. 101, n. 3, p. 191-199, 2011. Disponível em http://www.scielo.br/pdf/isz/v101n3/06.pdf

MOUTINHO, A. Serra da Estrela: Caracterização Ecológica De Lagoas Naturais. Dissertação (Mestrado em Ecologia, Ambiente e Território) - Faculdade de Ciências Universidade do Porto, Porto, 2016.

ROCHA, J.V.C. Análise Dos Impactos Ambientais Provocados Por Incêndios No Estado Da Califórnia De 1999 a 2017. Investigaciones Geográficas, v. 55, p. 147163, 2018.

SHWARTZ, A.; TURBÉ, A.; SIMON, L.; JULLIARD, R. Enhancing urban biodiversity and its influence on city-dwellers: An experiment. Biological Conservation, v. 171, p. 82-90, 2014. Disponível em https://doi.org/10.1016/j.biocon.2014.01.009

SILVA, F.A. Proposição de Metodologia em Suporte à Avaliação do Impacto do Uso e Ocupação do Solo na Desconformidade da Qualidade da Agua em Pequenas e Microbacias Hidrográficas Rurais. 2018. Dissertação (Mestrado em Engenharia Ambiental) - Universidade Federal Do Espírito Santo, Vitória,2018.

THORNHILL, I.; BATTY, L.; DEATH, R.G.; FRIBERG, N.R.; LEDGER, M.E. Local and landscape scale determinants of macroinvertebrate assemblages and their conservation value in ponds across an urban land-use gradient. Biodiversity and Conservation, v. 26, n. 5, p 1065-1086, 2017. Disponível em https://link.springer.com/article/10.1007/s10531-016-1286-4

USEPA. Evaluation of the Nation's Lakes: Field Operations Manual. EPA 841-B07-004. Washington D.C., E.S. Environmental Protection Agency. 332 p, 2007. 